PROCEEDINGS OF THE

AMERICAN MATHEMATICAL SOCIETY

Volume 128, Number 6, Pages 1613-1616

S 0002-9939(99)05482-9

Article electronically published on November 23, 1999

\title{
ON INTEGERS OF THE FORM $2^{k} \pm p_{1}^{\alpha_{1}} p_{2}^{\alpha_{2}} \cdots p_{r}^{\alpha_{r}}$
}

\author{
YONG-GAO CHEN \\ (Communicated by David E. Rohrlich)
}

\begin{abstract}
In this paper we prove that the set of positive odd integers which have no representation of the form $2^{n} \pm p^{\alpha} q^{\beta}$, where $p, q$ are distinct odd primes and $n, \alpha, \beta$ are nonnegative integers, has positive lower asymptotic density in the set of all positive odd integers.
\end{abstract}

\section{INTRODUCTION}

The problem of expressing an odd integer $m>1$ in the form $2^{n}+p$, where $p$ is a prime and $n$ is a nonnegative integer, is an old one. Romanoff [8] showed that the set of positive odd numbers which can be expressed in the form $2^{n}+p$ has positive asymptotic density in the set of all positive odd numbers. P. Erdős [ 6] exhibited a residue class of odd integers each of which has no representation of the form $2^{n}+p$. Cohen and Selfridge [5] proved that there exist (infinitely many ) odd numbers which are neither the sum nor the difference of a power of two and a prime power.

For a positive integer $n$ and an integer $a$ let

$$
a(n)=\{a+n k: k \in \mathbb{Z}\} .
$$

We call $\left\{a_{i}\left(n_{i}\right)\right\}_{i=1}^{t}$ a covering system if every integer $y$ satisfies $y \equiv a_{i}\left(\bmod n_{i}\right)$ for at least one value of $i$. For the construction of covering systems one may refer to S. L. G. Choi [4. For further related information one may see Guy [7, A19, B21 and F13.

For convenience we give the following definitions.

Definition 1. A positive integer $d$ is called a $(a, b)$-primitive divisor of order $n$ if $d \mid a^{n}-b^{n}$ and $d \not a^{m}-b^{m}$ for all $1 \leq m<n$.

Definition 2. $\left\{a_{i}\left(n_{i}\right)\right\}_{i=1}^{t}$ is called an $m$-covering system if every integer belongs to at least $m$ of $a_{1}\left(n_{1}\right), a_{2}\left(n_{2}\right), \cdots, a_{t}\left(n_{t}\right)$.

Definition 3. $\left\{a_{i}\left(n_{i}\right)\right\}_{i=1}^{t}$ is called a $(2,1)$-primitive $m$-covering system if $\left\{a_{i}\left(n_{i}\right)\right\}_{i=1}^{t}$ is an $m$-covering system and there exist distinct primes $p_{1}, p_{2}, \cdots, p_{t}$ such that, for each $i, p_{i}$ is a $(2,1)$-primitive divisor of order $n_{i}(1 \leq i \leq t)$.

For example 0(2),3(4),5(8),9(16), 17(32),33(64),1(64) is a $(2,1)$-primitive 1covering system (corresponding primes are 3, 5, 17, 257, 65537, 641, 6700417 respectively).

Received by the editors July 20, 1998 .

2000 Mathematics Subject Classification. Primary 11A07, 11B25.

This research was supported by the Fok Ying Tung Education Foundation and the National Natural Science Foundation of China, Grant No 19701015. 
From the form $\pm 2^{n} \pm p^{\alpha}$ to $\pm 2^{n} \pm p^{\alpha} q^{\beta}$ there are some essential difficulties. The first is to construct a $(2,1)$-primitive 2 -covering system. We employ Choi's method to complete such a construction. From this we need only consider $p, q$ in a finite set for our purpose. The second difficulty is to give additional conditions as in the proof of Theorem 2 of [2] or to do a similar thing. To avoid this we employ a result of Baker to show that for $p, q$ in a finite set, the set of integers of the form $\pm 2^{n} \pm p^{\alpha} q^{\beta}$ is very thin in the set of all positive odd integers. For later use we give the argument in a general form.

In this paper the following results are proved.

Theorem. Suppose that there exists a (2, 1)-primitive $r$-covering system. Then the set of odd positive integers which have neither the form $2^{n}+q_{1}^{\alpha_{1}} \cdots q_{r}^{\alpha_{r}}$ nor the form $2^{n}-q_{1}^{\alpha_{1}} \cdots q_{r}^{\alpha_{r}}$, where $q_{1}, q_{2}, \cdots, q_{r}$ are distinct positive odd primes and $n, \alpha_{1}, \cdots, \alpha_{r}$ are nonnegative integers, has positive lower asymptotic density.

Corollary. The set of odd positive integers which have neither the form $2^{n}+p^{\alpha} q^{\beta}$ nor the form $2^{n}-p^{\alpha} q^{\beta}$, where $p, q$ are distinct positive odd primes and $n, \alpha, \beta$ are nonnegative integers, has positive lower asymptotic density.

Remark 1. For given integers $a, b$ with $a b$ prime to corresponding primes $p_{1}, \cdots, p_{r}$ in Definition 3, the same conclusion is true when one replaces odd integers by the integers prime to $2 a b$ and one replaces $2^{n} \pm q_{1}^{\alpha_{1}} \cdots q_{r}^{\alpha_{r}}$ by $a 2^{n} \pm b q_{1}^{\alpha_{1}} \cdots q_{r}^{\alpha_{r}}$.

Remark 2. I believe that for every $r \geq 1$ there exists a $(2,1)$-primitive $r$-covering system.

\section{Proofs of the Theorem and the Corollary}

For the case with minus we need a result of A. Baker.

Let $\alpha_{1}, \cdots, \alpha_{n}$ be non-zero algebraic numbers. Let $K$ be their splitting field over $\mathbf{Q}$, and let $D=[K: \mathbf{Q}]$. We denote $A_{1}, \cdots, A_{n}$ upper bounds for the heights of $\alpha_{1}, \cdots, \alpha_{n}$ respectively, and assume that $A_{j} \geq 2$ for $1 \leq j \leq n$. Let

$$
\omega^{\prime}=\prod_{j=1}^{n-1} \log A_{j}, \quad \omega=\omega^{\prime} \log A_{n} .
$$

Lemma 1 (Baker 1]). There exist effectively computable absolute constants $c_{1}>0$ and $c_{2}>0$ such that the inequalities

$$
0<\left|\alpha_{1}^{b_{1}} \cdots \alpha_{n}^{b_{n}}-1\right|<\exp \left(-\left(c_{1} n D\right)^{c_{2} n} \omega \log \omega^{\prime} \log B\right)
$$

have no solutions in rational integers $b_{1}, \cdots, b_{n}$ with absolute values at most $B \geq 2$.

As a result of Lemma 1 we have

Lemma 2. Let $p_{1}, \cdots, p_{r}$ be given positive primes. Then the Diophantine inequality

$$
\left|2^{n}-p_{1}^{\alpha_{1}} \cdots p_{r}^{\alpha_{r}}\right| \leq x
$$

has at most $(2 \log x)^{r+1}$ nonnegative integral solutions $n, \alpha_{1}, \cdots, \alpha_{r}$ for $x \geq c$, where $c$ is a constant depending only on $p_{1}, \cdots, p_{r}$.

Proof. Suppose that $n, \alpha_{1}, \cdots, \alpha_{r}$ are not all zero. Let

$$
\delta=\left(c_{1}(r+1)\right)^{c_{2}(r+1)} \prod_{i=1}^{r} \log p_{i} \cdot \log 2 \cdot \log \prod_{i=1}^{r} \log p_{i} .
$$


By Lemma 1 we have

$$
\begin{aligned}
\left|2^{n}-p_{1}^{\alpha_{1}} \cdots p_{r}^{\alpha_{r}}\right| & =2^{n}\left|1-2^{-n} p_{1}^{\alpha_{1}} \cdots p_{r}^{\alpha_{r}}\right| \\
& \geq \frac{2^{n}}{\left(2 \max \left\{n, \alpha_{1}, \cdots, \alpha_{r}\right\}\right)^{\delta}}
\end{aligned}
$$

and

$$
\begin{aligned}
\left|2^{n}-p_{1}^{\alpha_{1}} \cdots p_{r}^{\alpha_{r}}\right| & =p_{1}^{\alpha_{1}} \cdots p_{r}^{\alpha_{r}}\left|2^{n} p_{1}^{-\alpha_{1}} \cdots p_{r}^{-\alpha_{r}}-1\right| \\
& \geq \frac{p_{1}^{\alpha_{1}} \cdots p_{r}^{\alpha_{r}}}{\left(2 \max \left\{n, \alpha_{1}, \cdots, \alpha_{r}\right\}\right)^{\delta}} .
\end{aligned}
$$

Thus

$$
\begin{aligned}
\left|2^{n}-p_{1}^{\alpha_{1}} \cdots p_{r}^{\alpha_{r}}\right| & =2^{n}\left|1-2^{-n} p_{1}^{\alpha_{1}} \cdots p_{r}^{\alpha_{r}}\right| \\
& \geq \frac{2^{\max \left\{n, \alpha_{1}, \cdots, \alpha_{r}\right\}}}{\left(2 \max \left\{n, \alpha_{1}, \cdots, \alpha_{r}\right\}\right)^{\delta}} .
\end{aligned}
$$

Then Lemma 2 follows immediately.

Proof of the Theorem. Suppose that $\left\{a_{i}\left(n_{i}\right)\right\}_{i=1}^{t}$ is a $(2,1)$-primitive $r$-covering system and $p_{1}, \cdots, p_{t}$ are corresponding primes in Definition 3 . Take an integer $M$ satisfying

$$
M \equiv 2^{a_{i}} \quad\left(\bmod p_{i}\right), \quad i=1, \cdots, t .
$$

For any positive integer $n$ there exist $i_{1}, \cdots, i_{r}$ with $1 \leq i_{1}<i_{2}<\cdots<i_{r} \leq t$ and

$$
n \in a_{i_{j}}\left(n_{i_{j}}\right), \quad j=1,2, \cdots, r .
$$

Then by (1) and

$$
2^{n_{i_{j}}} \equiv 1 \quad\left(\bmod p_{i_{j}}\right), \quad j=1, \cdots, r,
$$

we have

$$
M \equiv 2^{n} \quad\left(\bmod p_{i_{j}}\right), \quad j=1, \cdots, r
$$

Thus

$$
M=2^{n}+p_{i_{1}}^{\alpha_{i_{1}}} \cdots p_{i_{r}}^{\alpha_{i_{r}}} b, \quad \alpha_{i_{j}}>0(j=1,2, \cdots r), b \in \mathbb{Z} .
$$

Hence, if $M$ has the form

$$
M=2^{n} \pm q_{1}^{\beta_{1}} \cdots q_{r}^{\beta_{r}},
$$

where $q_{1}, \cdots, q_{r}$ are primes, then $q_{i} \in\left\{p_{1}, \cdots, p_{t}\right\}(i=1,2, \cdots r)$. It is clear that the number of integers $M$ with

$$
M=2^{n}+q_{1}^{\beta_{1}} \cdots q_{r}^{\beta_{r}} \leq x, \quad q_{i} \in\left\{p_{1}, \cdots, p_{t}\right\}, i=1,2, \cdots, r,
$$

is less than $c_{t}^{r}(2 \log x)^{r+1}$ for $x \geq X_{1}$. By Lemma 2 the number of positive integers $M$ with

$$
M=2^{n}-q_{1}^{\beta_{1}} \cdots q_{r}^{\beta_{r}} \leq x, \quad q_{i} \in\left\{p_{1}, \cdots, p_{t}\right\}, i=1,2, \cdots, r,
$$

is less than $c_{t}^{r}(2 \log x)^{r+1}$ for $x \geq X_{2}$. It is well known that the number of positive odd integers $M$ with (1) and $M \leq x$ is more than

$$
\frac{x}{2 p_{1} \cdots p_{t}}-1, \quad x \geq X_{3}
$$


Therefore, for $x \geq \max \left\{X_{1}, X_{2}, X_{3}\right\}$, there exist at least $\frac{x}{2 p_{1} \cdots p_{t}}-1-2 c_{t}^{r}(2 \log x)^{r+1}$ positive odd numbers $M \leq x$ which have neither the form $2^{n}+q_{1}^{\beta_{1}} \cdots q_{r}^{\beta_{r}}$ nor the form $2^{n}-q_{1}^{\beta_{1}} \cdots q_{r}^{\beta_{r}}$, where $q_{1}, \cdots, q_{r}$ are primes. This completes the proof of the Theorem.

Proof of the Corollary. Since

$$
A=\{0(2), 3(4), 5(8), 9(16), 17(32), 33(64), 1(64)\}
$$

and

$$
\begin{aligned}
B= & \{0(3), 4(9), 2(12), 10(18), 8(24), 16(36), 34(36), 20(48), 44(48), \\
& 1(5), 5(10), 3(15), 7(20), 4(25), 14(25), 13(30), 17(40), 9(50), \\
& 19(50), 23(60), 53(60), 37(80), 49(100), 77(120), 99(150), 157(160), \\
& 199(200), 199(300), 237(480), 157(240), 299(600)\}
\end{aligned}
$$

are both 1-covering systems, $A \cup B$ is a 2-covering system. By Birkhoff and Vandiver [3] (or Bang [2], Zsigmondy [9]), for $n \geq 2, n \neq 6$, there exist $(2,1)$-primitive prime divisors of order $n$. Again, 641, 6700417 are $(2,1)$-primitive prime divisors of order 64 ; write this as $64 \leftrightarrow 641,6700417$. Similarly, $36 \leftrightarrow 37,109 ; 48 \leftrightarrow 97,673$; $25 \leftrightarrow 601,1801 ; 50 \leftrightarrow 251,4051 ; 60 \leftrightarrow 61,1321$. Thus $A \cup B$ is a $(2,1)$-primitive 2 -covering system. Now the corollary follows from the theorem.

\section{REFERENCES}

1. A. Baker, The theory of linear forms in logarithms, Transcendence Theory: Advances and Applications (Academic Press, London and New York, 1977). MR 58:16543

2. A. S. Bang, Taltheoretiske Undersgelser, Tidsskrift for Mat. (5), 4(1886), 70-80, 130-137.

3. G. D. Birkhoff and H. S. Vandiver, On the integral divisors of $a^{n}-b^{n}$, Ann. Math. 5(1904), 173-180.

4. S. L. G. Choi, Covering the set of integers by congruence classes of distinct moduli, Math. Comput. 25(1971), 885-895. MR 45:6744

5. F. Cohen and J. L. Selfridge, Not every number is the sum or difference of two prime powers, Math. Comput. 29(1975), 79-81. MR 51:12758

6. P. Erdős, On integers of the form $2^{r}+p$ and some related problems, Summa Brasil. Math. 2(1947-51), 113-123. MR 13:437i

7. R. K. Guy, Unsolved Problems in Number Theory, 2nd ed., Springer, New York, 1994. MR 96e: 11002

8. N. P. Romanoff, Über einige Sätze der additiven Zahlentheorie, Math. Ann. 57(1934), 668-678.

9. K. Zsigmondy, Zur Theorie der Potenzreste, Monatsh. Math. 3(1892), 265-284.

Department of Mathematics, Nanjing Normal University, Nanjing 210097, People's Republic of China

E-mail address: ygchen@pine.njnu.edu.cn 